\title{
PERSONALIDAD, HÁBITOS DE ESTUDIO Y PREFERENCIAS POR GÉNEROS TELEVISIVOS EN JÓVENES UNIVERSITARIOS ${ }^{1}$
}

\author{
William Montgomery Urday ${ }^{2}$
}

Se recogen datos referentes a la interacción entre aspectos intelectuales y emocionales de la audiencia juvenil en relación con los efectos de la televisión. Por ello se aplicaron tres instrumentos para medir respectivamente los factores de personalidad, los hábitos de estudio y las preferencias por géneros de programación televisiva en 264 sujetos, con el fin de conocer y analizar tanto los perfiles generales como sus interrelaciones. Los resultados indican que hay diferencias significativas: a) en la personalidad de hombres y mujeres en los factores $\mathrm{O}$ y $\mathrm{Q}_{l}$ del test $16 f-a$, b) en los hábitos de estudio a favor de las mujeres, c) entre hombres y mujeres respecto a los hábitos de consumo televisivo, y también d) hay algunas relaciones significativas entre factores de personalidad, estrategias lectivas y consumo televisivo.

PALABRAS CLAVE: Personalidad, hábitos de estudio, hábitos de consumo televisivo.

It's very important to get facts of interaction between intellective and emotional aspects of young audience in connection with TV effects. In this work it's applied three tests for measurement of personality factors, study habits and televisive habits consume in 264 subjects, with the goal to know and to analyze your general profiles and relationships. The results to showed some mean differences in men and women on several topics, and some mean relations between personality factors, study strategies and televisive consume.

KEY WORDS: Personality, study habits, televisive habits consume.

\footnotetext{
${ }^{1}$ Texto adaptado de la investigación «Factores de personalidad, hábitos de estudio y preferencias por géneros de programación televisiva en jóvenes universitarios de Letras» (I1PSI, UNMSM, 2002). Responsable: W. Montgomery. Miembros: C. Velásquez, G. Guevara y H. Hernández. Colaboradores: A. Grajeda, D. Tarazona, S. Espejo y M. Vásquez.

${ }^{2}$ Docente de la Facultad de Psicología UNMSM. Correo electrónico: williamonty@ hotmail.com.
} 


\section{INTRODUCCION}

La televisión ha invadido instituciones, hogares y conciencias, dando forma (o ayudando a darla) a un estilo de vida que caracteriza nuestros tiempos. La alta capacidad de la tecnología audiovisual para formar impresiones rápidas y duraderas en los individuos, sobre todo aquellos en proceso de formación, es notoria (Cebrián, 1998). Se dice que en los países desarrollados la televisión es, después del trabajo y del descanso, la tercera actividad más frecuente para los adultos, y la segunda para los estudiantes después del sueño. La importancia de dicho problema se puede ilustrar cuantitativamente. De acuerdo con datos del año 1992 (Ander-Egg, 1996), sobre la base de la información procedente de cincuenta países desarrollados, subdesarrollados, capitalistas y socialistas, se ha constatado que aproximadamente había en el mundo 873 millones de televisores y un promedio de por lo menos dos personas frente a cada uno de ellos, dedicándose entre tres a cuatro horas diarias (lo cual equivale a 900 o 1400 horas semanales) a ver los programas televisivos. Eso significa que, ya en ese entonces, la humanidad en conjunto invertían seis millones de horas diarias en semejante actividad. Cada día vería televisión en promedio: a) el $90 \%$ de los individuos de 5 a 15 años de edad, b) e185\% de aquellos mayores de 15 años, y c) el $95 \%$ de personas de 65 años a más, aumentando conforme avanza la vejez. Los niños, antes de entrar a la escuela, verían entre tres mil a cinco mil horas diarias la televisión. Asimismo, si un niño comenzó a ver televisión a los tres años, al llegar a los 65 ya habría visto entre 60 mil a 75 mil horas (vale decir casi el 12\% o 15\% de su vida). Por último, hasta los 17 años, el adolescente habría visto un promedio de cuatro horas y media de violencia a la semana, y más de 18 mil «muertes» simuladas.

De un tiempo a esta parte tales datos posiblemente se hayan incrementado $\mathrm{y}$ correlacionado con el descalabro moral y cultural que conlleva la difusión de valores alienantes e ideologías deshumanizadas, característica de las sociedades de consumo bajo el modelo neoliberal. Se necesita recoger más información precisa referente a los efectos e interacciones que la televisión tiene respecto a los receptores de sus mensajes, especialmente sobre los niños y los jóvenes.

En nuestro medio el nivel de confianza de los jóvenes limeños entre 15 y 24 años (hombres y mujeres sin diferencias significativas) en la televisión es muy superior a la depositada en los demás medios, como lo muestra una encuesta de CEDRO (Castro de la Mata y Rojas, 1998), ascendiendo hasta el $75.6 \%$ contra $30.8 \%$ de confianza en la radio y el $16.9 \%$ en diarios y revistas. Los temas televisivos preferidos son las películas y comedias $(38.3 \%)$, noticieros y programas políticos $(36.2 \%)$, telenovelas $(30.8 \%)$, deportes (15.3\%), programas cómicos (13.3\%), series (11.2\%), dibujos animados (9\%) y ciencia y cultura (6.6.). Las mujeres brindan especial atención a las telenovelas y los hombres a los deportes y películas. Otra encuesta de nota, llevada a cabo por el grupo de Al faro y Macassi (1995) con muestras de padres de niños de 6-11 años, muestra resultados parecidos en la audiencia paterna de tales géneros, y en la universidad, García Toledo (2000) diseñó y aplicó una encuesta de opinión y dos guías de observación sobre consumo televisivo a 200 estudiantes de la Facultad de Educación, encontrando también preferencias generales por las teleseries (45.5\%), las revistas informativas (39\%) y las telenovelas $(31.5 \%)$, con similares diferencias entre hombres y mujeres como en los anteriores casos. Todos esos resultados parecen indicar una continuidad en los gustos televisivos de las personas a través de un largo período adolescencia-juventud-adultez temprana.

Lo dicho lleva a fijarse en cómo se formaría la personalidad del receptor de los mensajes de la televisión, que, de acuerdo con las teorías de los «efectos fuertes» (por ejemplo Noelle Neumann, 1993, Le.) y «sutiles» (véase McCoombs 1985, Le.), constituyen un ambiente envolvente y perpetuo cuyas influencias directas e indirectas no pueden eludirse. 
Lleva a fijarse también en las estrategias de cómo selecciona la información y cómo aprende el sujeto (sus hábitos de estudio) dentro de ese ambiente.

\section{Personalidad y Consumo Televisivo}

Respecto a la personalidad del receptor promedio en el proceso de lo que podría considerarse formación básica en el desarrollo, la estructura conformada históricamente como estilo interactivo del individuo (Ribes y Sánchez, 1990) se va consolidando hasta convertirse en una variable que afecta las interacciones presentes y futuras. Los patrones culturales, los valores, las creencias y las costumbres, vale decir, la estimulación social y emocional de mayor impacto sobre el comportamiento se refleja en la personalidad, que extiende su influjo a todos los planos de la vida del individuo a través de la modulación y regulación tanto de su forma de pensar como de sentir y reaccionar (actitudes). Staats (1997, t.e.) llama «repertorios conductuales básicos» a los aprendidos durante la niñez, indicando que son primordiales a la hora de la evaluación psicológica, pues dan amplias pistas sobre el resto de la conducta en relación con diversos sucesos, objetos y personas

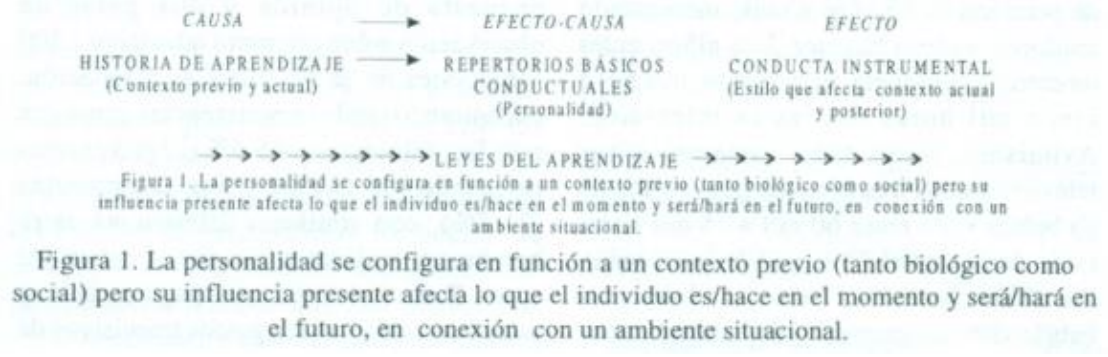

En la indagación de los efectos de la TV sobre la audiencia, e inversamente, la conducta de la audiencia frente a la TV, los investigadores han tratado de hallar regularidades en los efectos de los medios sobre las personas: tendencias predecibles y evidencia estadística para documentar el modo en que se producen. De acuerdo con Biaggi (1999, t.e.), ya en 1929 H. Blumer realizó una encuesta adolescente para determinar si los recuerdos infantiles acerca de películas vistas influían en su conducta actual, concluyendo que sÍ. En 1939, tras la supuesta «invasión marciana» fraguada desde la radio por Orson Welles, H. Cantril enfatizó la existencia de diferencias personales como variables mediadoras entre el auditor y el medio, lo que fue corroborado por numerosos estudios posteriores, como los hechos por el Departamento de Salud, Educación y Bienestar (TV and Social Behavior, de 1969) y el Instituto Nacional de Salud Mental de los Estados Unidos (de 1982). Ésta última investigación arrojó las siguientes conclusiones principales: a) hay correlación directa entre la violencia transmitida por TV y el comportamiento agresivo, pero no se puede predecir quién será afectado y porqué, b) quienes ven con mayor frecuencia la TV son más temerosos, desconfiados y aprehensivos que los que la ven esporádicamente, y c) los niños que ven programas socialmente constructivos tienden a actuar más responsablemente.

También se destaca en nuestro medio la aproximación a los efectos que sobre la tele audiencia infanta-juvenil tienen los contenidos de la programación televisiva en cuanto a la conformación de actitudes violentas (Palma, 1993). Aquí se analizan el aprendizaje social y las implicancias de los modelos simbólicos en cuanto a la imitación, la internalización de valores, expectativas y creencias de los individuos sobre la base de un cuestionario construido para el caso, concluyendo que existe una relación directamente proporcional entre la tendencia personal violenta y la observación de ciertos contenidos audiovisuales. Otro trabajo descriptivo-comparativo entre sujetos de educación secundaria y superior 
mayores de 17 años se llevó a cabo utilizando como instrumentos una encuesta sobre el tiempo dedicado a ver TV, una ficha socioeconómica y un registro de comportamientos frente a la televisión, identificando un alto grado de teledependencia (Luna, 1993). Mares (1996, t.e.) por su parte demuestra que, en el marco de dicha teledependencia, la actividad de ver programas de ficción y de información se relaciona positivamente con los juicios confusos que los individuos tienen sobre la realidad social.

\section{Personalidad y Hábitos de Estudio}

Según León (1996) la televisión aporta una experiencia concreta, dinámica y asociativa que controla al sujeto con imágenes cuyo contenido hipnotiza y fascina. Por el contrario, la lectura conlleva una actitud concentrada y esforzada por codificar, elaborar y activar inferencias de conocimiento previo. La televisión promueve el pensamiento impulsivo, fragmentado y poco persistente, emotivo y basado en impresiones de agrado-desagrado, mientras que la lectura promueve el pensamiento racional y reflexivo, basado en opiniones de acuerdo-desacuerdo. Semejantes diferencias pueden hacer distante la congruencia entre hábitos de estudio y consumo televisivo si hay un desbalance en el tiempo dedicado a cada una de esas actividades, o en cuanto a su calidad.

Aunque suele verificarse que una mayor exposición televisiva no necesariamente va de la mano con un menor rendimiento académico, la interacción del consumo de TV con alguna otra variable sí parece ser influyente (ver por ejemplo Mendoza, 1999). En general parece que el consumo televisivo produce, más en la niñez que en la adolescencia, un aumento de la repetición memorística y se interpone en el aprendizaje escolar, amén de adormecer la recepción crítica (Quiróz, 1989) y afectar la lectura de libros deteriorando las actitudes favorables hacia ellos y disminuyendo la concentración (Coostra y Van Deer Root, 1996, t.e.). Por otro lado Connelly, Kirstin, Dubois y Stanley (1998), midiendo conductas de estudio como marcar palabras o párrafos, tomar notas, etc.; en una muestra aleatoria de 30 alumnos, hallaron diferencias entre aquellos que tienen calificaciones más altas y los que tienen las más bajas. Eso respecto a la manera como los primeros manejan su tendencia a ensoñar o distraerse durante las clases, y dedican más tiempo al estudio. Tales resultados muestran, indirectamente, que si la televisión induce desconcentración o cansancio visual y a la vez resta horas lectivas a las utilizadas por un individuo es un efectivo obstáculo a la consolidación de su repertorio académico.

La Universidad de Montemorelos montó un proyecto para investigar los hábitos de estudio de sus alumnos y aspectos psicológicos relacionados (locus de control, inteligencia, tiempo dedicado al trabajo académico, etc.). La muestra fue de 1108 estudiantes de pre y postgrado (54\% mujeres), resultando del cuestionario a qué fueron sometidos que el $75 \%$ de ellos declaran tener alguna conducta in apropiada, como por ejemplo la de olvidar las notas de clase apenas tomada una prueba y nunca más volver a repasar los contenidos evaluados (44\%). El $42 \%$ estudia con la idea de recordar solamente hasta que termina el examen, y en general sólo un $15 \%$ se ubica como personas con un definido propósito de aprender (Grajales, 2002).

Si bien los hábitos de estudio no constituyen productos determinados únicamente por los factores de personalidad, y frecuentemente en el ámbito universitario no presentan correlaciones claras con ellos (Ponce, 1988 y Aliaga, 1998 con el Inventario de Cattell; Salas y Vera, 1999; con el Inventario de Guilford-Zimmerman); Velásquez (1998) en cuanto al comportamiento psicosocial; Enriquez S 1999 en cuanto al autoconcepto y la ansiedad; Tapia (1994) y Aliaga. Ponce, Gutiérrez, Díaz, Reyes y Pinto (2001), a través de la revisión de diversas investigaciones se nota que siempre subsiste la sospecha de que, por 
lo menos en algunos campos, sí existe interacción funcional, sea en forma general (Bruckmann, 1976; Cornejo, 1988; Ponce, 1988; Jara, 1991; Badiljo, 1991) o específica (Velásquez, 1998; Enríquez, 1999; Aliaga, Ponce, Gutiérrez, Díaz, Reyes y Pinto, 2001). Lo cierto es que los hábitos de estudio, como capacidades prácticas de «actividad intelectual» especialmente importante en la especialización profesional, al ser formas aprendidas de conducirse académicamente y orientar a orientaciones intelectivas particulares, deben ser influidos por -y a la vez influenciar a- otras estructuras del comportamiento (Montgomery, 2000). El estilo interactivo particular de la personalidad incluye aspectos intelectivos que de todas maneras juegan un papel en las estrategias de búsqueda y asimilación de cierto tipo de informaciones en relación con objetivos de logro (De Raad \& Schouwenburg, 1996). Inversamente los hábitos de estudio, en tanto forman repertorios de disciplina intelectual-afectiva e incrementan las competencias del individuo, afectan su personalidad (Ponce, 1988; Badillo, 1991; Jara, 1991), como lo demuestra también un trabajo de Blickle (1996), quien mediante un análisis factorial constató que las relaciones entre los rasgos de la personalidad y los grados básicos de desarrollo fueron mediadas por las estrategias para aprender.

Dentro de las competencias aludidas se hallan las vinculadas a la observación de programas televisivos. Algunos de esos efectos pueden entreverse a través de escasos estudios recientes que relacionan la comunicación familiar, el rendimiento académico y el tiempo de exposición a la TV (por ejemplo Mendoza, 1999), siendo importante su detección con el fin de hallar maneras efectivas de formar una recepción crítica (Orozco, 1991) y mejorar también la formación de la personalidad en general.

Sobre la base de las razones señaladas, se considera de relevancia estudiar las interacciones entre tres de las variables que afectan el comportamiento individual de los estudiantes universitarios: el sistema factorial de la personalidad, los patrones habituales de aptitud académica metacognitiva o hábitos de estudio, y el grado de preferencia hacia emisiones de los medios de comunicación social. El presente trabajo se halla en esa línea, planteando:

1. ¿Cuáles son las dimensiones de personalidad predominantes entre los ingresantes a la Facultad de Letras?

2.¿Cuál es su nivel de hábitos de estudio?

3.¿Cuáles son sus preferencias por géneros de programación televisiva?

4.¿Cuáles son las relaciones entre los factores de personalidad, los hábitos de estudio y las preferencias por géneros de programación televisiva?

En base a ello, los objetivos generales son los siguientes:

- Explicitar las dimensiones de personalidad predominantes entre los ingresantes a la Facultad de Letras.

- Conocer el nivel general de sus hábitos de estudio.

- Conocer sus preferencias por géneros de programación televisiva.

- Conocer y analizar las relaciones entre los factores de personalidad, los hábitos de estudio y las preferencias por géneros de programación televisiva de los ingresantes a la Facultad de Letras.

\section{MÉTODO}


Diseño de la investigación. Corresponde al descriptivo correlacional, con una sola muestra, de acuerdo con el siguiente diagrama:

\section{Muestra}

M = Estudiantes ingresantes a la Facultad de Letras de la UNMSM

Participantes. Se aplicaron los instrumentos a una muestra de 264 sujetos entre 16-24 años, de ambos sexos, de un universo total de 323 ingresantes a las especialidades de Filosofía, Comunicación Social, Lingüística.

\section{Variable}

$\mathrm{Ox}=\ll$ Factores de personalidad» $\mathrm{Oy}=\ll$ Hábitos de estudio»

$\mathrm{Oz}=\ll$ Preferencias por géneros de programación $\mathrm{TV} »$

Literatura, Arte y Bibliotecología en la Facultad de Letras y Ciencias Humanas de la UNMSM. La distribución de la muestra por escuelas figura en la tabla siguiente:

Tabla 1. Distribución muestral total y parcial según E.A.P.

\begin{tabular}{|l|l|l|l|l|}
\hline $\begin{array}{c}\text { Escuela distribución } \\
\text { muestral }\end{array}$ & $\begin{array}{c}\text { Numero } \\
\text { de sujetos }\end{array}$ & $\begin{array}{c}\text { Edad } \\
\text { promedio }\end{array}$ & Hombres & Mujeres \\
\hline Arte & 36 & 19.39 & 18 & 18 \\
Bibliotecología & 37 & 18.84 & 15 & 23 \\
Comunicación Social & 71 & 18.56 & 30 & 41 \\
Filosofía & 38 & 19.18 & 24 & 14 \\
Lingüística & 52 & 18.70 & 12 & 40 \\
Literatura & 30 & 19.80 & 17 & 13 \\
Total & 264 & 19.07 & 116 & 148 \\
\hline
\end{tabular}

\section{Instrumentos}

Inventario de Personalidad 16 PF de R.B. Catell (1982, t.e.), compuesto por 16 escalas de carácter primario que miden diferentes factores de la personalidad (187 ítemes). Para la presente muestra se determinó en 0.70 el índice de confiabilidad de la prueba. Los rótulos de cada factor se han redenominado de acuerdo con la información de Carver y Scheier (1997, t.e.):
A. Reserva-Calidez
B. Concreción-Abstracción
C. Inestabilidad-Estabilidad
E. Deferencia-Dominancia
F. Formalidad-Vivacidad
G. Despreocupación-Escrupulosidad
H. Timidez-Audacia
I. Utilitarismo-Sensibilidad
L. Confianza-Vigilancia
M. Practicidad-Imaginación
N. Franqueza-Astucia
O. Seguridad-Aprehensión
$\mathrm{Q}_{1} \quad$ Tradición-Apertura
$\mathrm{Q}_{2} \quad$ Dependencia-Autosuficiencia 
$\mathrm{Q}_{3} \quad$ Descuido-Autoperfección

$\mathrm{Q}_{4}$ Relajación-Tensión

\section{Inventario de Hábitos de Estudio}

CASM-85 de L. Vicuña (1993), compuesto de cinco escalas que miden diferentes aspectos relacionados con las estrategias de estudio a un nivel de confiabilidad del 0.86.

I.¿Cómo estudia usted? (12 ítemes)

II. ¿Cómo hace sus tareas? (10 ítemes)

III. ¿Cómo prepara sus exámenes? (11 ítemes)

IV. ¿Cómo escucha las clases? (12 ítemes)

V. ¿Qué acompaña sus momentos de estudio? (6 ítemes)

Escala Dunn-Rankin de 4 puntos denominada «Encuesta de Preferencias por Género Televisivo», elaborada para computar preferencias de consumo por doce géneros de programación televisiva (Nunca, Algunas veces, Casi siempre, Siempre) haciendo notar que el protocolo incluye ejemplos populares para cada uno, extraídos de la programación de TV abierta en la época en que se llevó a cabo la medición. Los géneros considerados son los siguientes:

Informativo

Ciencia y técnica

Deportes

Opinión

Arte popular

Series y Películas
Espectáculo

Arte especializado

Religión

Publicidad

Hogareño

Novelas

\section{RESULTADOS}

Las tablas 2,3 y 4 muestran respectivamente los perfiles de personalidad, hábitos de estudio y preferencias por géneros de TV. Las tablas 5, 6 y 7 muestran las diferencias de medias $(P=0.05)$ entre hombre y mujeres en las tres variables estudiadas. A su vez, las tablas 7,8 y 9 indican las correlaciones entre dichas variables en sus distintas dimensiones.

Tabla 2. Perfiles de personalidad en la muestra estudiada
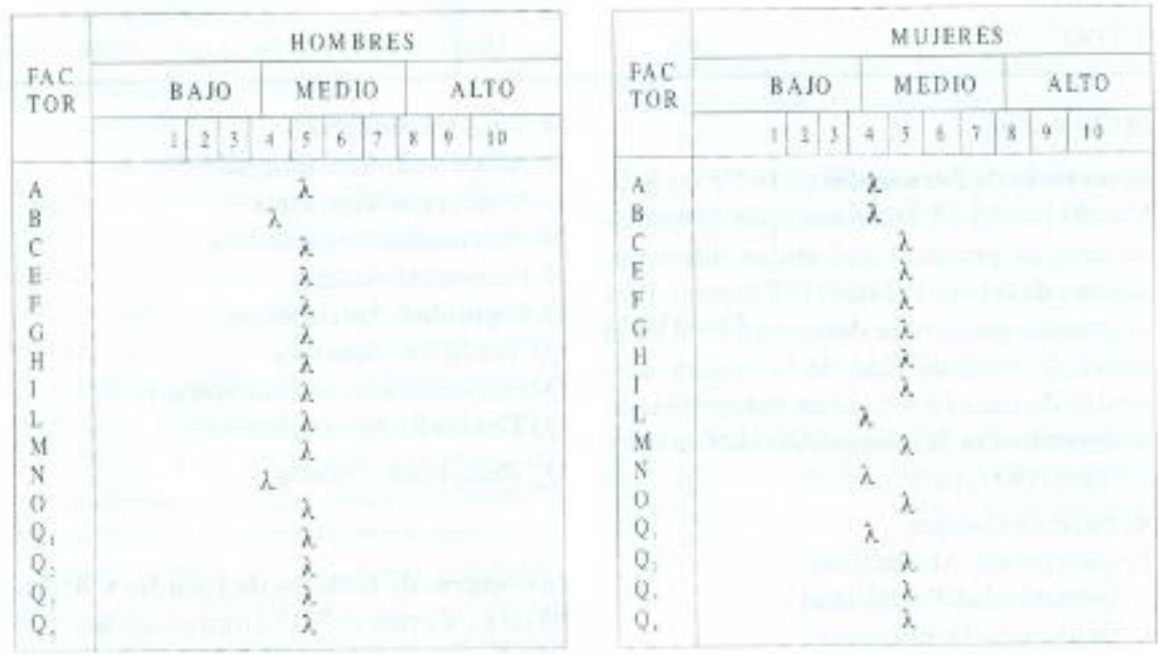
Tabla 3. Perfiles de hábitos de estudio en la muestra estudiada.

\begin{tabular}{|c|c|c|c|c|c|c|c|c|c|c|c|c|}
\hline \multirow{2}{*}{ Fac. } & \multicolumn{4}{|c|}{ HOMBRFS } & \multirow[b]{2}{*}{$\mathrm{B}$} & \multirow[b]{2}{*}{$\mathrm{MB}$} & \multicolumn{6}{|c|}{ MUJERES } \\
\hline & $\mathrm{MM}$ & $\mathrm{M}$ & B- & $\mathrm{B}+$ & & & MM & $\mathrm{M}$ & B- & $\mathrm{B}+$ & $\mathrm{B}$ & MB \\
\hline I & & & & 7.83 & & & & & & & 8.64 & \\
\hline II & & & & 6.97 & & & & & & 7.80 & & \\
\hline III & & & & 7.36 & & & & & & 8.20 & & \\
\hline IV & & & & 7.06 & & & & & & 7.68 & & \\
\hline V & & & & 5.53 & & & & & & 5.44 & & \\
\hline
\end{tabular}

Tabla 3.Perfiles de preferencias por GTV en la muestra estudiada.

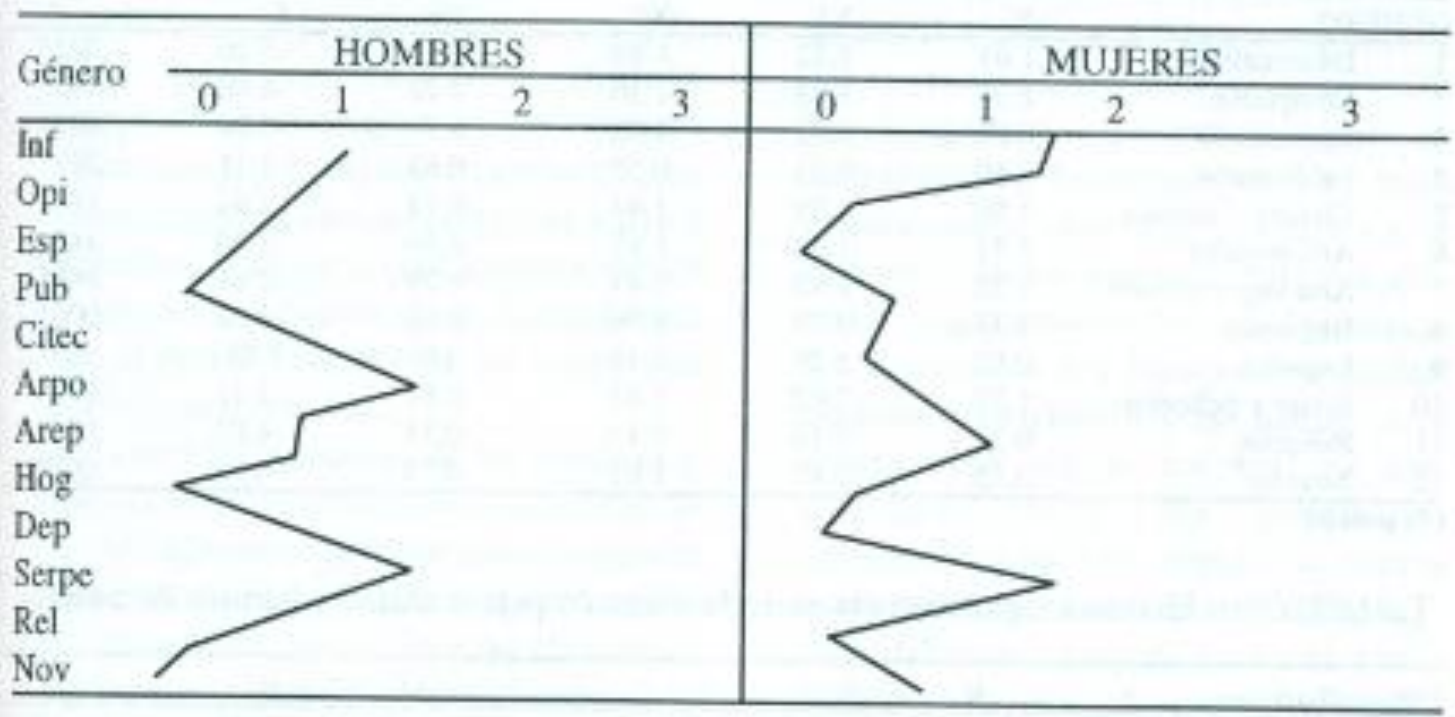

Tabla 4. Diferencia de medias $(X)$ entre hombres $\left(X_{1}\right)$ y mujeres $\left(X_{2}\right)$ en Factores de Personalidad.

\begin{tabular}{|c|c|c|c|c|c|c|}
\hline FACTOR & $\mathrm{x}$ & $\mathrm{XI}$ & $\mathrm{X}_{2}$ & DS & $t$ & $P$ \\
\hline A Reserva-Calidez & 9.27 & 9.13 & 9.37 & 2.44 & -802 & .423 \\
\hline B Concreción-Abstracción & 3.85 & 3.96 & 3.76 & 1.62 & 964 & .336 \\
\hline C Inestabilidad-Estabilidad & 12.72 & 13.03 & 12.47 & 3.01 & 1.49 & .139 \\
\hline E Deferencia-Dominancia & 13.31 & 13.41 & 13.23 & 2.93 & .505 & .614 \\
\hline F Formalidad-Vivacidad & 13.00 & 13.30 & 12.78 & 3.40 & 1.25 & .213 \\
\hline G Despreocup-Escrupulosidnd & 10.97 & 10.81 & 11.10 & 2.50 & -.939 & 349 \\
\hline H Timidez-Audacia & 13.29 & 13.50 & 13.12 & 3.11 & 980 & 328 \\
\hline I Utilitarismo-Sensibilidad & 12.15 & 12.39 & 11.96 & 2.70 & 1.28 & .202 \\
\hline L. Confianza-Vigilancia & 9.30 & 9.62 & 9.05 & 2.65 & 1.73 & .085 \\
\hline M Practicidad-Imaginación & 13.10 & 13.16 & 13.05 & 2.97 & 315 & 753 \\
\hline N Francqueza-Astucia & 13.12 & 12.90 & 13.30 & 2,87 & -1.13 & 261 \\
\hline o Seguridad-Aprebensión & 12.77 & 12.35 & 13.10 & 3.06 & -1.98 & $048^{*}$ \\
\hline Q, Tradición-Apertura & 10.84 & 11.21 & 10.56 & 2.48 & 2.12 & $035^{*}$ \\
\hline Q, Dependencia/Autosuficienc. & 10.24 & 10.26 & 10.23 & 2.92 & .080 & 937 \\
\hline Q, Descuado-Autoperfección & 9.98 & 10.10 & 9.88 & 2.65 & 683 & 495 \\
\hline Q, Tensión-Relajación & 14.35 & 14.57 & 14.17 & 2.93 & 1.10 & 274 \\
\hline
\end{tabular}


Tabla 5. Diferencia de medias entre hombres $\left(X_{1}\right)$ y mujeres $\left(X_{2}\right)$ en hábitos de estudio (factores y puntaje total).

\begin{tabular}{|c|c|c|c|c|c|c|}
\hline FACTOR & $\mathrm{x}$ & $\mathrm{xl}$ & $\mathrm{X2}$ & DS & 1 & $P$ \\
\hline 1. Cómo estudiar & 8. 28 & 7.83 & 8.64 & 2.25 & -2.93 & $.0 \times 4^{*}$ \\
\hline 11. Cómo hacer tareas & 743 & 6.97 & 7.80 & 2.09 & -3.27 & $001 *$ \\
\hline III. Preparación de exámenes & 7.83 & 3.36 & 8.20 & 2.27 & -3.01 & $.003^{*}$ \\
\hline IV. Fscuchar clases & 3.41 & 7.06 & 7.68 & 2.41 & -2.09 & $037^{*}$ \\
\hline V. Acomp, momeatos de est. & 5.48 & 5,53 & 5.44 & 1.57 & 4.90 & 625 \\
\hline Pustaje Total & 36,43 & 34.75 & 37.75 & 7.90 & -3.11 & $0,02^{\circ}$ \\
\hline
\end{tabular}

(x) p<0.05

Tabla 6. Diferencia de medias entre hombres $\left(\mathrm{X}_{1}\right)$ y mujeres $\left(\mathrm{X}_{2}\right)$ en preferencias por géneros televisivos

\begin{tabular}{|c|c|c|c|c|c|c|c|}
\hline \multicolumn{2}{|c|}{ GENERO } & \multirow{2}{*}{$\frac{x}{1.67}$} & \multirow{2}{*}{$\frac{X 1}{1.52}$} & \multirow{2}{*}{$\frac{\mathrm{x} 2}{1.80}$} & \multirow{2}{*}{$\frac{\mathrm{DS}}{0.72}$} & \multirow{2}{*}{$\frac{t}{-3.20}$} & \multirow{2}{*}{$\frac{P}{09 / 2^{x}}$} \\
\hline 1. & Informativo & & & & & & \\
\hline 2. & De opiaión & 1.36 & 1.43 & 1.30 & 0.78 & -1.40 & .165 \\
\hline 3. & Espectáculo & 0.97 & 0.83 & 1.08 & 0.74 & -2.80 & $.005^{*}$ \\
\hline $4{ }^{\circ}$ & Publicitario & 0,50 & 0.45 & 0.53 & 0.62 & -1.11 & 269 \\
\hline 5. & Ciencia y técnica & 1.50 & 1.59 & 1.44 & 0.74 & -1.61 & 109 \\
\hline 6. & Arte popular & 1.31 & 1.23 & 1.37 & 0.80 & -1.40 & .162 \\
\hline 7. & Arte especializado & 1.32 & 1.17 & 1.43 & 0.79 & -2.68 & $.008^{*}$ \\
\hline 8. & Hogareino & 0.37 & 0.13 & 0.56 & 0.62 & -6.02 & $.000^{*}$ \\
\hline 9. & Deportes & 0.83 & 1.29 & 0.46 & 0.95 & 7.84 & $.000^{\circ}$ \\
\hline 10. & Series y peliculas & 1.74 & 1.62 & 1.83 & 0.81 & -2.11 & $.036^{\circ}$ \\
\hline 11. & Religión & 0.31 & 0.16 & 043 & 0.55 & -4.07 & $.000^{*}$ \\
\hline 12. & Novelas & 0.75 & 0.41 & 1.01 & 0.75 & -6.97 & $.000^{*}$ \\
\hline
\end{tabular}

Tabla 7. Correlaciones significativas entre factores de personalidad y hábitos de estudio.

\begin{tabular}{lllllllll}
\hline Hábitos/Factores & $\mathrm{A}$ & $\mathrm{B}$ & $\mathrm{C}$ & $\mathrm{I}$ & $\mathrm{L}$ & $\mathrm{M}$ & $\mathrm{N}$ & $\mathrm{Q3}$ \\
\hline I & .023 & & & .006 & & & .000 \\
II & .001 & .002 & .003 & & & 001 & .005 \\
III & .022 & .012 & .008 & & & & .007 & 005 \\
IV & & .046 & .001 & .000 & 010 & 017 & .002 & .005 \\
V & & & & & & & & \\
TOTAL & .002 & .003 & .001 & .003 & .042 & .002 & .000 \\
\hline
\end{tabular}

Tabla 8. Correlaciones significativas entre factores de personalidad y preferencias por géneros televisivos.

\begin{tabular}{|c|c|c|c|c|c|c|c|c|}
\hline GénerovFat & A & B & $\mathbf{F}$ & G & $\mathrm{L}$ & $\mathrm{N}$ & $\mathrm{QI}$ & Q3 \\
\hline $\ln f$ & 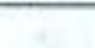 & & & & & .024 & & \\
\hline Opi & & .015 & & & & & & 048 \\
\hline Esp & & & 027 & .046 & & & & \\
\hline Arp & $\cos 7$ & & 022 & & & & .008 & \\
\hline Arep & .020 & & & & & & .003 & \\
\hline Serpe & & .029 & .047 & & 014 & & .004 & \\
\hline Nor & & & & .007 & .013 & & & \\
\hline
\end{tabular}


Tabla 9. Correlaciones significativas entre hábitos de estudio y preferencias por géneros televisivos.

\begin{tabular}{lllllllll}
\hline Hábitos/Géneros & $\operatorname{lnf}$ & Opi & Esp & Citec & Arp & Arep & Hog & Dep \\
\hline I & .000 & .001 & & .001 & .002 & .000 & & \\
II & .000 & .002 & .005 & .000 & & & & .003 \\
III & .013 & & & .001 & .009 & .012 & .022 & .005 \\
IV & .000 & .004 & & .000 & & .017 & & .005 \\
V & & & & & & & & .011 \\
TOTAL & .000 & .002 & .008 & .000 & .005 & .000 & & \\
\hline
\end{tabular}

$\mathbf{t}$

\section{CONCLUSIONES}

1. Existen diferencias significativas entre las medias de hombres y mujeres en dos de los factores de la personalidad, ( $\mathrm{O}$ Y Q1). Los puntajes en ellos indican respectivamente que: a) las mujeres tienen un nivel más alto de ansiedad y de sentimiento de menor aceptación, y b) los hombres ostentan mayor apertura mental y tolerancia a nuevas ideas. Sin embargo, en el perfil ninguna de las tendencias excede el promedio.

Los perfiles generales de los hombres y de las mujeres muestran dos tendencias moderadamente predominantes: una a la lentitud de comprensión y aprendizaje e inclinación hacia interpretaciones concretas y literales, y otra a la sencillez, espontaneidad y poca sofisticación. Las mujeres también presentan moderados rasgos de carácter reservado y socialmente escéptico, precisión y rigidez en criterios y acciones. Se inclinan al conservadurismo y a no ser competitivas. Los perfiles de comparación de sexos por escuelas denotan los siguientes rasgos: en Comunicación Los hombres muestran un nivel mayor de inteligencia sin salir del promedio. Hay una fuerte tendencia femenina y masculina a la suspicacia y a la despreocupación. Las mujeres se inclinan al pensar práctico y a la timidez/ susceptibilidad. En Bibliotecología hombres y mujeres tienden a la autosuficiencia y a la reserva y poca abstracción. Los hombres son más adaptables y seguros, en tanto que las mujeres son más realistas. En Filosofía hay una marcada tendencia masculina a la con fiabilidad, y femenina a la escrupulosidad y a la integración social, a la reserva y a la inestabilidad emocional. En Lingüística se ven rasgos de dominancia, despreocupación, baja abstracción, dureza, franqueza y seguridad en los varones, mientras que en las mujeres una tendencia a la reserva. En Literatura los hombres muestran elevada dependencia y las damas poca integración social. En Arte no hay puntajes fuera del promedio, sobresaliendo sin embargo cierta tendencia masculina a la ansiedad.

2. Hay diferencias significativas entre hombres y mujeres en cuanto a los hábitos de estudio, favorable a las últimas. El perfil general de hábitos de estudio muestra una tendencia positiva en hombres y mujeres, pero aquellas muestran una significativa mejor aptitud en todos los rubros comprendidos, con excepción de uno. Así, en los factores 1, I1, III Y IV las mujeres declaran subrayar los puntos más importantes, repasar, buscar significados en el diccionario, relacionar lo leído con otros contenidos, preguntar para aclaraciones, llevar un estudio diario regular y preparar los temas con anticipación en mayor medida que los hombres. En el factor V no hay mayor diferencia entre sexos. Las diferencias de medias entre escuelas indican que la muestra de Comunicación tiene el máximo nivel de hábitos de estudio, contrastando significativamente con las escuelas de Arte, Filosofía y Bibliotecología. En segundo lugar está Linguiística, que también muestra diferencias significativas con Bibliotecología, la escuela con menor índice de hábitos de estudio. 
3. Hay diferencias significativas en la preferencia por géneros de programación televisiva en cuanto a la visión de informativos, de programas de espectáculo, de arte especializado, hogareño, de series y películas, programas religiosos y de novelas, a favor de las mujeres (lo que indica una ligeramente mayor audiencia femenina de TV), y también una diferencia significativa mayor en cuanto a deportes a favor de los hombres. El perfil general de preferencias por géneros de programación televisiva muestra en hombres una mayor opción por las series y películas, ciencia-técnica e informativos, y una menor por los géneros hogareño, religioso y publicitario. Las mujeres también se inclinan en primer lugar por las series y películas, pero ponen en segundo lugar lo informativo y en tercero el género de ciencia y técnica. Se inclinan menos por ver programas religiosos, deportivos y publicitarios.

4. Hay algunas relaciones significativas entre factores de personalidad y preferencias por géneros de programación televisiva, tales como que el factor $A$ cruza con las preferencias por los géneros de Arte popular y Arte especializado. El factor $B$ hace contacto con el género de Opinión, lo que indicaría que hay una tendencia de «recepción fácil» con el fin de orientarse de acuerdo con interpretaciones ajenas. A su vez hay una correlación negativa con Series y Películas, posiblemente porque involucran un tipo de recepción considerado más complejo (por ejemplo entender el argumento, los personajes, etc.). La dimensión $F$ se correlaciona negativamente con los géneros de Espectáculo, Arte Popular y Series y Películas. Eso puede interpretarse como que a mayor prudencia, seriedad, introspección y represión en el carácter, hay un menor interés por géneros posiblemente considerados distracciones frívolas. La dimensión $G$ se relaciona significativamente con los géneros de Espectáculo y Novelas, lo cual muestra que la evitación de normas y obligaciones y la falta de compromisos de grupo se concilia con la opción por la frivolidad y el melodramatismo simplón. El factor $L$ tiene relación negativa con Series y Películas y Novelas. A mayor adaptabilidad y no competitividad, entonces, correspondería menor opción por esos géneros. Esto podría explicarse considerando que el meollo argumental de dichos tipos de programas es justamente pautado por la competitividad, los celos y hasta la envidia entre los personajes. La dimensión $N$ se correlaciona con el género de Información. Ello sugeriría, dados los rasgos que distinguen a dicho factor, que la credulidad juega un papel. La persona, mientras más simple y espontánea es, más cree sin cuestionar lo que se le dice en los programas informativos. El factor $Q 1$ casa negativamente con Arte Popular, Arte Especializado y Series y Películas, lo que no es extraño teniendo en cuenta que éstos géneros suelen contener propuestas a veces avanzadas y a veces chocantes con la ideología o la moral predominantes. La dimensión $Q 3$ relaciona con el género de Opinión. Siendo el rasgo típico de dicho factor de personalidad la falta de preocupación por ceñirse o aceptar normas sociales, su virtual sensación de desajuste puede aliviarse disintiendo, manifestándose rebelde o viendo cómo se «rebelan» otros contra ciertos eventos.

5. Hay relaciones significativas entre algunos factores de personalidad y Hábitos de estudio, tales como que el factor $A$ se correlaciona con casi el total de factores de Hábitos de estudio, lo que concilia con su actividad de trabajar en solitario y su búsqueda de precisión. Aunque no muestra correlación explícita con el factor $\mathrm{V}$, las relaciones significativas de la dimensión $A$ con la preferencia por los diversos géneros de Arte indicaría que, más allá del reporte verbal, sí hay el acompañamiento citado. La dimensión $B$ easa también con el total de Hábitos de estudio, y con II, III y IV, que suponen hasta cierto punto hábitos mecánicos y de probable escucha pasiva. El factor $\mathrm{C}$ muestra con toda razón una correlación altamente negativa con los hábitos de estudio, dado que hace imposible concentrarse en tareas lectivas. El factor $\boldsymbol{I}$ hace contacto con I, IV Y el total, 
denotando que se va sólo a lo juzgado más importante de la actividad de estudio en cuanto a la adquisición del conocimiento. La dimensión $L$ se relaciona con el total de los Hábitos de estudio, aunque con ninguno en particular más que los otros. El factor $M$ iguala con los hábitos II y IV, así como con el total. Tal como en 1, se juzga esas actividades como las más importantes, pero esta vez al parecer en términos de mostrar el conocimiento ya adquirido. La dimensión $N$ contacta con el total y con I, II, III y IV . Esto, si se tienen en cuenta también el factor $L$ ya reseñado, indicaría fuerte convencionalismo. Es decir que el ejercicio de Hábitos de estudio se concibe simplemente como una actividad rutinaria y obligatoria. Finalmente, el factor $Q I$ se relaciona con el factor IV lo que, teniendo en cuenta un parágrafo anterior (preferencia por el género de Opinión), se explicaría por la compensación de mostrarse cuestionador ante ciertas normas.

6. Hay diferencias significativas hábitos de estudio y preferencias por géneros de programación televisiva, tales como que el género de Información contacta con el total y con I, II, III y IV El género de Opinión se relaciona con el total y con I, II y IV. El género de Espectáculo correlaciona negativamente con el total y con II, lo que da a entender que se consideran incompatibles. El género de Ciencia y Técnica hace contacto con el total y con I, II, III y IV El género de Arte Popular contacta con el total y con I, II y III. El género de Arte Especializado casa con el total y con I y II. El género Hogareño se relaciona con III. El género de Deportes con el total y con II.

\section{DISCUSIÓN}

Observando los perfiles generales de personalidad se nota mucha homogeneidad entre hombres y mujeres, lo que no es sorpresa ya que se había anticipado en la hipótesis respectiva que sólo en ciertos factores de personalidad (no explicitados por la falta de datos previos al respecto) podrían encontrarse diferencias significativas, los cuales fueron $\mathrm{O}$ (mayor en mujeres) y Q1 (mayor en hombres). Esto introduce elementos nuevos con respecto a investigaciones anteriores (Cornejo, 1988; Ponce, 1988), que no detectaron diferencias significativas entre sexos.

En cuanto a las preferencias por géneros de programación televisiva hay mayores sorpresas, pues si bien investigaciones anteriores ya muestran que la visión de programas de entretenimiento mediante teleseries y películas y la afición por los programas informativos son alternativas favoritas de la juventud (Castro de la Mata y Rojas, 1998; García Toledo, 2000), no confieren lugares preferentes en cambio a la ciencia y a la técnica, género que en el presente estudio sí ocupa el tercer lugar (segundo entre los varones). A su vez las telenovelas y deportes son relegados de su tercer y cuarto lugar en otras investigaciones con muestras ajenas a Letras, a modestos octavo y noveno lugares entre doce en el presente trabajo. Persisten, sin embargo, las diferencias significativas en éstos géneros en varones (mayor en deportes) y damas (mayor en telenovelas)3. Sorprende en cierto modo también el más explícito interés manifestado por las mujeres por los géneros de series y películas e informativos, aunque está de acuerdo con la tendencia mundial de preferencias hacia la ficción (Ferrés, 1995).

En cuanto al nivel de hábitos de estudio, éste se muestra como bastante aceptable en los ingresantes a Letras, denotando que ellos saben teóricamente qué hacer respecto a cada uno de los elementos que permitirían un mejor rendimiento lectivo. Especialmente las mujeres, que se distinguen significativamente de los varones en este rubro. La tradición investigativa anterior decía que: a) la tendencia de hábitos inadecuados era bastante elevada (hasta 49.24 $\%$ 4: véase Aguilar, 1972), y b) no había diferencias significativas entre sexos (Bruckman, 
1976).

La correlación entre factores de personalidad y hábitos de estudio ofrece algunas coincidencias con lo hallado en estudios anteriores. Así, asumiendo la equivalencia de dos de las dimensiones del Inventario de J. Pinillos (Estabilidad emocional y Sinceridad) -en que Ponce (1988) encontró relaciones significativas con los hábitos de estudio-, respectivamente con los factores e y $N$ del $16 \mathrm{PF}$, se nota que los resultados son similares. La influencia de la dimensión Neuroticismo-Estabilidad, adaptada del Test de Personalidad de Eysenck por Bruckman (1976) y los indicadores emocionales del Inventario de Temperamento de Guilford-Zimmerman hallados por Salas y Vera (1999) en sus muestras de estudio también se consideran en los mismos términos. Por otra parte, el trabajo de Aliaga y co15. (2001) con la prueba de Cattell enfatiza el rasgo 1 come frecuentemente covariante con el rendimiento académico, lo que casa con las relaciones significativas encontradas en el presente trabajo. Algo más preocupante -sobre todo en ingresantes a un programa de Letras- es la relación hallada entre promedios de un buen nivel de hábitos lectivos y una relativamente baja capacidad e interés intelectual, como los conseguidos por la muestra general que es materia de este análisis (en el análisis por escuelas Filosofía Comunicación y Literatura se eximen de ese cargo).

Algunos comentarios se pueden hacer respecto a la relación entre personalidad) preferencias por géneros televisivos. Uno de ellos es que definitivamente ciertos rasgos parecen influir sobre la elección de ciertos tipos de programas, y probablemente éstos últimos desarrollarían (o debilitarían) más $1<$ fuerza de los rasgos. Según se ve a través de los resultados obtenidos hay varia: tendencias más definidas que otras, guiada: fundamentalmente por los usos gratificaciones (ecuación «expectativa de recompensa/esfuerzo requerido»: Schramn (1986, trad. esp.; p. 16) que cada individuo ejerce o encuentra en su propio consumo de televisión. Esto apoya el obvio hecho de que hay una audiencia «activa», pero siempre en contacto con una realidad envolvente dada por el ambiente de interacción simbólica especialmente proveído por la TV Otro comentario que surge es la falta de recepción crítica, confirmando dudas casi permanentes sobre la interacción sujeto-medio masivo (Quiróz, 1989), pues los géneros de Información y de Opinión se ven respectivamente ligados a factores como el $N$ y el $\mathrm{Q} I$ en ni veles de tendencia relativamente baja. Es decir, mucha sencillez, poco cuestionamiento profundo de los contenidos y si mucha importancia al afán, al parecer artificial mente promovido por los medios, de no ceñirse a normas (idénticamente a como 10 haría un «niño caprichoso»).

Los resultados sobre las relaciones entre hábitos de estudio y preferencias por géneros televisivos son un primer indicador empírico de sus nexos directos. Por ejemplo, es posible confrontar con los datos objetivos la tan difundida creencia de que el consumo televisivo produce tendencias a la ensoñación en clases y a la desconcentración lectiva en general (Coostra y Van Deer Root, 1996, trad. esp.; Connelly et al, 1998). A este respecto, puede notarse que en particular son géneros como el deportivo y el de espectáculo (de fuerte correlación negativa con el total de hábitos de estudio, especialmente con el factor TI), los que alejan al estudiante de su labor o se oponen a ella. Otro aspecto final resaltante es que aunque no hay un reconocimiento explícito de los sujetos, a través de sus respuestas al cuestionario CASM-85, del factor V, sí se infiere una gran influencia de cosas como esa en las correlaciones altamente significativas de los hábitos de estudiar y preparar exámenes con los géneros Arte Popular y Arte Especializado (especialmente la música de videoclips). 


\section{REFERENCIAS BIBLIOGRÁFICAS}

Alfaro, R. y Macassi, S. (1995). Seducidos por la tele: Huellas educativas de la televisión en padres y niños. Lima: Calandria.

Aguilar, B. (1972). Hábitos de estudio de alumnos universitarios. Tesis Lic. Psicología. UNMSM, Lima

Aliaga, J. (1998). La ubicación espontánea del asiento en el aula como función de la inteligencia, la personalidad, el rendimiento académico y el sexo. Revista Peruana de Psicología. 4(7-8), 117-140.

Aliaga, J. et al (2001). Variables psicológicas relacionadas con el rendimiento académico en matemática y estadística en alumnos del primer y segundo año de la Facultad de Psicología de la UNMSM. Revista de Investigación en Psicología. 4(1), 35-52.

Ander-Egg, E. (1996). Teleadictos y vidiotas en la aldea planetaria: ¿Qué hace la TV con nosotros? Buenos Aires: Lumen.

Badillo, L.G. (1991). Estudio descriptivo correlacional de los hábitos de estudio y las dimensiones de la personalidad en alumnos del I ciclo de la Facultad de Educación. Tesis Lic. Psicología UIGV, Lima.

Baggaley, J.P. y Duck, S.w. (1979, trad. esp.). Análisis del mensaje televisivo. Barcelona: Gustavo Gili.

Biaggi, Sh. (1999). Impacto de los medios. Una introducción a los medios masivos de comunicación. México: International Thomson Editores.

Blickle, G. (1996). Personality traits, learning strategies and performance. European Journal of Personality 10(5), 337-352.

Bruckmann, E. (1976). Correlación entre capacidad intelectual, hábitos de estudio y rendimiento académico de los alumnos de la escuela Nacional de Bibliotecarios. Tesis Lic. Psicología UNMSM, Lima.

Carver, Ch. S. y Scheier, M.F. (1997, trad. esp.). Teorías de la personalidad. México: Prentice-Hall.

Castro de la Mata, R. y Rojas, M. (1998). Los jóvenes en el Perú: Opiniones, actitudes y valores. Monografía de investigación. Lima: CEDRO.

Catell, R.B. (1982, trad. esp.). l6PF. Cuestionario de Personalidad. Madrid: TEA.

Cebrián, M. (1998).Información televisiva: Mediaciones, contenidos, expresión y programación. Madrid: Síntesis.

Coolstra, C.M. y Van Deer Root, T.H. (1996, trad. esp.). Efectos longitudinales de la televisión sobre los hábitos de lectura de los niños. Human Communication Research, 23(1), 4-35. Abstract disponible en: ehu.es/zer/zen2/ abstracting.html/

Connally, O.F., Kirstin, P., dubois, N.F. \& Stanley, R. (1998, Abril 15). Structured interview study of the long-term effects of a college study skills course: Traces and selfreport measures. Ponencia a la reunión Anual de la American Educational Research Association. San Diego, California.

Cornejo, N. (1988). NeuroticismoEstabilidad, actitudes y habilidades de estudio en jóvenes universitarios de costa y sierra. Tesis de Doctorado en Letras. UNMSM, Lima.

De Raad, B. \& Schouwenburg, H.C. (1996). Personality in learning and education. European Journal of Personality 10(5), 303-336.

Enríquez, J.L. (1999). El autoconcepto y la ansiedad ante los exámenes como predictores del rendimiento académico en estudiantes de secundaria. Revista de Psicología de la UICV. III (1-2), 149169.

Eysenck, H.J. (1979, t.e.). Fundamentos biológicos de la personalidad. Barcelona: Fontanella.

Ferrer, E. (1997). Información y comunicación. México: FCE. 
Ferrés, J. (1995) Televisión, espectáculo y educación. Comunicar, 4, 37-41.

García Toledo (2000). El consumo de televisión. Lima: Autor.

Grajales, T. (2002, t.e.) Hábitos de estudio de estudiantes universitarios. Centro de Investigación Educativa. Artículo disponible en http://grajales.net/ habitosdeestudio. pdf

Jara, W. (1991). Relación entre las dimensiones básicas de la personalidad y los hábitos de estudio en alumnos de la Facultad de Psicología de la UICY. Tesis Lic. Psic. UIGV, Lima.

Luna, E. (1993, octubre). Conductas compatibles con la adicóón a la TV. Ponencia al VI Congreso Nacional de Psicología. Lima, Perú.

Mares, M.L. (1996, t.e.). El papel de la confusión entre fuentes en la incubación de la televisión sobre juicios de la realidad social. Human Communication Research, 23(1), 278-297. Abstract disponible en: ehu.es/zer/zen2/abstracting.html/

McCoombs, M.E. (1985, trad. esp.). La comunicación de masas en las campañas políticas: Información, gratificación y persuasión. En M. Moragas (Ed.). Sociología de la comunicación de masas (pp. 361-380). Barcelona: Gustavo Gili.

Mendoza, 1. (1999). Clima familiar, exposición televisiva y rendimiento escolar. Tesis Lic. en Comunicación Social. UNMSM, Lima.

Montera, V. (1993, octubre). ¿Ausencia de paradigmas o miedo a la libertad? ¿Cómo educar a través de los medios de comunicación? Ponencia al VI congreso Nacional de Psicología. Lima, Perú.

Montgomery, W. (2000). Cómo potenciar el trabajo intelectual a través de una conducta proactiva de salud, bienestar y orden personales. En W. Montgomery, W. Capa y H. Montes de Oca (Eds.). Análisis de la conducta: Nuevos enfoques, aplicaciones $e$ investigaciones (pp. 111133). Lima: ASPPSI.

Noelle-Neumann, E. (1993, t.e.). La espiral del silencio. Madrid: Alianza.

Orozco, G. (1991). La audiencia frente a la pantalla. Diálogos de la Comunicación, 30,5463.

Palma, S. (1993, octubre). Análisis de los contenidos de violencia en la programación televisiva peruana. Ponencia al VI congreso Nacional de Psicología. Lima, Perú.

Ponce, C. (1988). Estudio comparativo sobre hábitos de estudio y su relación con la inteligencia y la personalidad en grupos de ingresantes a la UNMSM. Tesis de Doctorado en Letras. UNMSM, Lima.

Ponce, C. (1992). Investigación transcultural sobre los hábitos de estudio en grupos de estudiantes universitarios peruanos y españoles. Theorema, 1 (2), p. 21.

Quiroz, M.T. (1989).¿Es posible hablar de una recepción crítica de TV? Dia-Lagos de la comunicación, 24, 117-125.

Ribes, E. y Sánchez, S. (1990). El problema de las diferencias individuales: Un análisis conceptual de la personalidad. En E. Ribes: Psicología general (pp. 231253). México: Trillas.

Sartori, G. (1999). Homo videns. Barcelona: Oikos Tau.

Schramm, W. (1986, t.e.). Cómo funciona la comunicación. En C. Fernandez y G.L. Dahnke (Eds.). La comunicación humana: Ciencia social (pp. 1-25). México: McGrawHill.

Shibutani, T. (1971, trad. esp.). Sociedad y personalidad. Buenos Aires: Paidós.

Staats, A.W. (1979). Conductismo social. México: El Manual Moderno.

Staats, A.W. (1997). Conducta y personalidad. Conductismo psicológico. Bilbao: Descleé de Brouwer.

Velásquez, C. (1998). Desajustes del comportamiento psicosocial del estudiante de la UNMSM. Revista de Investigación en Psicología. 1(2), 167-182.

Vicuña, L. (1993). Inventario de Hábitos de Estudio (CASM-85). Lima: Autor. 
Wolf, M. (1994). Los efectos sociales de los media. Barcelona: Paidós.

Zarzurí, R. (2002). Jóvenes y televisión: Un estudio sobre la cultura televisiva de los jóvenes. (44 pp.). Centro de Estudios Socioculturales. Artículo disponible en cesc.cl/cdv/pdf/n2.pdf 